\title{
Dynamic Total Cost of Ownership Optimiza- tion for IPTV Service Provider
}

\author{
P. Goudarzi", M. Adeli ${ }^{+}$, M.M. Azadfar ${ }^{*}$ and F. Ayatollahi \\ 'Multimedia Group, IT Faculty, Iran Telecom Research Center, Tehran-Iran \\ ${ }^{+}$Islamic Azad University of Dezful \\ Email: pgoudarzi(wi)itrc.ac.ir
}

Please use the following format when citing this chapter:

Goudarzi, P., Adeli, M., Azadfar, M.M., Ayatollahi, F., 2007, in IFIP International Federation for Information Processing. Volume 256, Ilome Networking, Al Agha. K.. Carcelle, X., Pujolle, G., (Boston: Springer). pp. 295-312. 


\title{
1 Introduction
}

\author{
P. Goudarzi ${ }^{*}$, M. Adeli ${ }^{+}$, M.M. Azadfar ${ }^{*}$ and F. Ayatollahi \\ • Multimedia Group, IT Faculty, Iran Telecom Research Center, Tehran-Iran \\ ${ }^{+}$Islamic Azad University of Dezful \\ Email: pgoudarzi@uitrc.ac.ir
}

\begin{abstract}
Total Cost of Ownership (TCO) for developing communication services comprises from two parts; CAPital EXpenditure (CAPEX) and OPerational EXpenditure (OPEX). These two types of costs are interrelated and affect any service provider's deployment strategy. In many traditional methods, selection of critical elements of a new service is performed in a heuristic manner aimed at reducing only the OPEX part of the TCO which is not necessarily optimal. In the current chapter, a brief review of the TCO for a communication service is proposed.
\end{abstract}

Keywords-TCO, CAPEX, OPEX

Total cost of ownership (TCO) is a financial estimate designed to help consumers and enterprise managers assess direct and indirect costs commonly related to software or hardware. It is a form of full cost accounting. Middleware and other systems needed to provide video are also part of the total CAPEX. In a business case, CAPEX can be broken into fixed and variable parts; fixed being those costs to build the requisite system and infrastructure to deliver the services, and variable being those costs incurred 
with individual subscriber take rates. CPE and in home installation are considered variable costs, along with DSL line cards, since the CAPEX is incurred only when service is taken. Ideally, fixed CAPEX should be minimized since it is the "at risk" investment to enter into the business. Variable CAPEX, although directly related to actual service take rate and revenue, cannot be so excessive as to present a ROI (Rate of Investment) that it creates unacceptable ROI.

IPTV business cases as well as actual deployments have shown that the in home CPE and installation costs amount to $60 \%$ or more of the total installed cost for the IPTV system [1].

With CPE and in-home installation representing the largest portion of total installed cost, it is the area best targeted for cost reduction.

OPEX is composed of funds used by a company to acquire or upgrade physical assets such as property, industrial buildings or equipment.

This type of outlay is made by companies to maint-ain or increase the scope of their operation. These expenditures include everything from repairing a roof to building a brand new factory.

Video-on-Demand (VoD) is the next killer-app and a subset of the IPTV service. Initial trials have been well received by customers and network operators are deploying VoD to increase subscriber revenues and service profitability. VoD allows subscribers to request the programming of their choice, when they want where they want it. It is this flexibility that appeals to the broader customer base when compared with regularly scheduled network programming of broadcast video.

In most traditional methods, the only objective is to minimize the OPEX part of the TCO by selecting critical components of the service in a heuristic manner. But, this approach may not necessarily result in optimal solution for the service providers.

For example, in deploying the IPTV service in Iran, the service providers select the number of the required edge servers in order to minimize the OPEX part of the TCO [2].

Because of its static nature, this method doesn't consider the interrelations between OPEX and CAPEX which varies with time. For example, though choosing a specific initial number of edge servers may be optimal at the first stages of service deployment, this may not lead to an optimal solution for TCO minimization problem as time elapses.

Any solution for minimizing the TCO must take into the account the dynamic characteristics of the problem as time elapses.

In the current work, a mathematical approach is developed to minimize the 
TCO. The proposed method tracks the dynamic changes in the number of subscribers and takes into the account the subscribers' geographical distributions and time. 


\title{
2 IPTV service
}

\author{
P. Goudarzi ${ }^{*}$ M. Adeli ${ }^{+}$, M.M. Azadfar ${ }^{*}$ and F. Ayatollahi \\ • Multimedia Group, IT Faculty, Iran Telecom Research Center, Tehran-Iran \\ + Islamic Azad University of Dezful \\ Email: pgoudarzi(c)itrc.ac.ir
}

\begin{abstract}
In the current chapter, an introduction about the basic components of an IPTV service scenario is developed. Then the various components delved in modeling of its TCO (including CAPEX and OPEX parts) are introduced.
\end{abstract}

Keywords-TCO, IPTV, CAPEX, OPEX

Total cost of ownership modeling is a tool that systematically accounts for all costs related to an IT investment decision. TCO models were initially developed by Gartner Research Corporation in 1987 and are now widely accepted. Simply stated, TCO includes all costs, direct and indirect, incurred throughout the life cycle of an asset, including acquisition and procurement, operations and maintenance, and end-of-life management.

TCO analysis originated with the Gartner group in 1987 and has since been developed in a number of different methodologies and software tools. A TCO assessment ideally offers a final statement reflecting not only the cost of purchase but all aspects in the further use and maintenance of the equipment, device, or system considered. This includes the costs of train- 
ing support personnel and the users of the system, costs associated with failure or outage (planned and unplanned), diminished performance incidents (i.e. if users are kept waiting), costs of security breaches (in loss of reputation and recovery costs), costs of disaster preparedness and recovery, floor space, electricity, development expenses, testing infrastructure and expenses, quality assurance, boot image control, marginal incremental growth, decommissioning, e-waste handling, and more.

When incorporated in any financial benefit analysis TCO provides a cost basis for determining the economic value of that investment.

The TCO concept is widely used in the automobile industry. In this context, the TCO denotes the cost of owning a vehicle from the purchase, through its maintenance, and finally its sale as a used car. Comparative TCO studies between various models help consumers choose a car to fit their needs and budget. In [2] after extensive market research, an estimation about the CAPEX and OPEX of each IPTV component is derived from which we have developed a mathematical model for the TCO of IPTV service in Section III.

IPTV describes a system where a digital television service is delivered using the Internet Protocol over a network infrastructure, which may include delivery by a broadband connection [4].

For residential users, IPTV is often provided in conjunction with VoD and may be bundled with Internet services such as Web access and VoIP. The commercial bundling of IPTV, VoIP and Internet access is referred to as a Triple Play. Adding the mobile voice service leads to the Quadruple Play denomination.

IPTV is typically supplied by a broadband operator using a closed network infrastructure. This closed network approach is in competition with the delivery of TV content over the public Internet. This type of delivery is widely called TV over Internet or Internet Television.

In businesses, IPTV may be used to deliver television content over corporate LANs and business networks. Perhaps a simpler definition of IPTV would be television content that, instead of being delivered through traditional formats and cabling, is received by the viewer through the technologies used for computer networks.

Broadcast IPTV has two major architecture forms: free and fee based. This sector is growing rapidly and major television broadcasters worldwide are transmitting their broadcast signal over the Internet. IPTV channels require only an Internet connection and an Internet enabled device such as a personal computer, iPod, HDTV connected to a computer or even a $3 \mathrm{G}$ cell/mobile phone to watch the IPTV broadcasts. 
A typical IPTV scenario is depicted in the Figure 1.

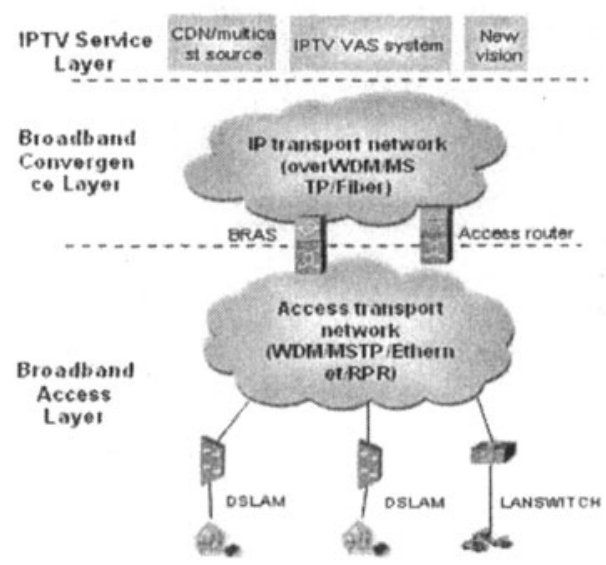

Figure 1. Typical IPTV service scenario

The basic components of IPTV service are, video streaming servers [5], edge streaming servers used for load balancing purposes, encoded content, transport and access QoS-enabled networks, BRAS (Broadband Remote Access Server), DSLAM (Digital Subscriber Line Access Multiplexer), STB (Set Top Box) and ADSL (Asymmetric DSL) modems.

Each IPTV component is associated with an incurred CAPEX and OPEX. Some components such as transport network are out of the service provider's control and impose only a long-term OPEX on the service provider's deployment strategy and some of the components such as content only consist of an initial CAPEX and don't impose any important OPEX on the service development.

In the following section, mathematical models for the CAPEX and OPEX of each IPTV component are developed and based on the proposed models; a dynamic solution for the TCO minimization problem is introduced. 


\title{
3 Problem Formulation
}

\author{
P. Goudarzi ${ }^{*}$ M. Adeli ${ }^{+}$, M.M. Azadfar ${ }^{*}$ and F. Ayatollahi \\ *Multimedia Group, IT Faculty, Iran Telecom Research Center, Tehran-Iran \\ ${ }^{+}$Islamic Azad University of Dezful \\ Email: pgoudarzicuitrc.ac.ir
}

\begin{abstract}
In this chapter based on a project which was performed in the Iran Telecom Research Center, a mathematical model is introduced which can model the CAPEX and OPEX parts of the IPTV'S TCO dynamically in terms of time and the number of service subscribers.
\end{abstract}

Keywords-TCO, IPTV, CAPEX, OPEX

In order to minimize the TCO (CAPEX+OPEX) of an IPTV service, we have developed in a heuristic manner, the mathematical models associated with the CAPEX and OPEX of each component which is involved in the service.

DSLAM, STB, ADSL modem, Edge Server, Main server are among the devices which together build an IPTV solution. In this section, mathematical models for CAPEX and OPEX of these components are presented. To do so, we first make an estimation of the initial cost of each component individually based on the research accomplished in [2]. The initial cost of 
STB, ADSL modem, DSLAM, BRAS, main server, edge server, content and infrastructure are denoted by $x_{\mathrm{STB}}, x_{\mathrm{MDM}}, x_{\mathrm{DSLM}}, x_{\mathrm{BRAS}}, x_{\mathrm{MSRVR}}$, $x_{\text {ESRVR }}, x_{\mathrm{CNT}}, x_{\text {INSTR }}$ respectively.

Table 1 shows the initial cost of the devices normalized by the initial cost of ADSL modem.

Table 1. Initial cost of IPTV devices normalized by ADSL modem cost

\begin{tabular}{llll}
\hline DEVICE & COST & DEVICE & COST \\
\hline$x_{\mathrm{STB}}$ & 3.33 & $x_{\mathrm{MSRVR}}$ & 176 \\
$x_{\mathrm{MDM}}$ & 1 & $x_{\mathrm{ESRVR}}$ & 44.44 \\
$x_{\mathrm{DSLM}}$ & 128 & $x_{\mathrm{CNT}}$ & 11.11 \\
$x_{\mathrm{BRAS}}$ & 10 & $x_{\text {INSTR }}$ & 100 \\
\hline
\end{tabular}

It must be mentioned that the above normalized values in Table (1) are obtained from a survey on the prices of each IPTV component which was investigated about the Iran's IT market in [2].

In order to cover the dynamic nature of the mentioned components of the TCO, the mathematical models for CAPEX and OPEX are chosen to be functions of time $t$, number of subscribers $n$ and number of edge servers $m$.

In the following equations, parameters beginning with ' $\mathrm{C}$ ' represent CAPEX and those beginning with ' $O$ ' represent OPEX.

The CAPEX and OPEX are modeled as follows:

$$
\begin{aligned}
& \mathrm{C}_{\mathrm{STB}}(n, t)=\left(x_{\mathrm{STB}}+\left(y_{\mathrm{STB}}-x_{\mathrm{STB}}\right) \mathrm{e}^{-z_{\mathrm{STB}} n}\right) . \\
& \left(2-e^{-e_{0} t}\right) \\
& \mathrm{O}_{\mathrm{STB}}(n, t)=x_{\mathrm{STB}}\left(1-\mathrm{e}^{-\mathrm{e}_{1} n}\right)\left(1-\mathrm{e}^{-\mathrm{c}_{2^{\prime}}}\right) \\
& \mathrm{C}_{\mathrm{MDM}}(n, t)=\left(x_{\mathrm{MDM}}+\left(y_{\mathrm{MDM}}-x_{\mathrm{MDM}}\right) \mathrm{e}^{-z_{\mathrm{MJM}} n}\right. \\
& \left(2-e^{-e_{0} t}\right) \\
& \mathrm{O}_{\mathrm{MDM}}(n, t)=x_{\mathrm{MDM}}\left(1-\mathrm{e}^{-\mathrm{c}_{1} n}\right)\left(1-\mathrm{e}^{-\mathrm{e}_{2} t}\right) \\
& \mathrm{C}_{\mathrm{DSLM}}(t)=x_{\mathrm{DSLM}} \cdot\left(2-e^{-e_{0} t}\right) \\
& \mathrm{O}_{\mathrm{DSLM}}(n, t)=x_{\mathrm{DSLM}}\left(1-\mathrm{e}^{-\mathrm{e}_{1} n}\right)\left(1-\mathrm{e}^{-\mathrm{e}_{2} t}\right)
\end{aligned}
$$




$$
\begin{gathered}
\mathrm{C}_{\mathrm{BRAS}}(t)=x_{\mathrm{BRAS}} \cdot\left(2-e^{-e_{0} t}\right) \\
\mathrm{O}_{\mathrm{BRAS}}(n, t)=\mathrm{x}_{\mathrm{BRAS}}\left(1-\mathrm{e}^{-\mathrm{e}_{1} n}\right)\left(1-\mathrm{e}^{-\mathrm{c}_{2} t}\right) \\
\mathrm{C}_{\mathrm{MSRVR}}(t)=x_{\mathrm{MSRVR}} \cdot\left(2-e^{-e_{0} t}\right) \\
\mathrm{O}_{\mathrm{MSRVR}}(n, t)=x_{\mathrm{MSRVR}}\left(1-\mathrm{e}^{-\mathrm{e}_{2} t}\right) \\
\mathrm{C}_{\mathrm{ESRVR}}(t)=x_{\mathrm{ESRVR}} \cdot\left(2-e^{-e_{0} t}\right) \\
\mathrm{O}_{\mathrm{ESRVR}}(n, t)=x_{\mathrm{ESRVR}}\left(1-\mathrm{e}^{-\mathrm{e}_{1} n}\right)\left(1-\mathrm{e}^{-\mathrm{e}_{2} t}\right) \\
\mathrm{C}_{\mathrm{CNT}}=x_{\mathrm{CNT}} \\
\mathrm{O}_{\mathrm{INSTR}}=\left(x_{\mathrm{INSTR}}+y_{\mathrm{INSTR}} \mathrm{e}^{-z_{\mathrm{INSTR}} m}\right) . \\
\left(p_{\mathrm{INSTR}}-q_{\mathrm{INSTR}} \mathrm{e}^{-s_{\mathrm{INSTR}} n}\right) . \\
\left(v_{\mathrm{INSTR}}-w_{\mathrm{INSTR}} \mathrm{e}^{-u_{\mathrm{INSTR}} t}\right)
\end{gathered}
$$

Where we have [6]:

$$
\begin{aligned}
& y_{\mathrm{STB}}=0.8 x_{\mathrm{STB}} ; y_{\mathrm{MDM}}=0.8 x_{\mathrm{MDM}} ; \\
& y_{\mathrm{INSTR}}=0.11 y_{\mathrm{NSTR}} ; e_{1}=3.22 \times 10^{-7} ; e_{2}=0.0053 ; \\
& z_{\mathrm{STB}}=z_{\mathrm{MDM}}=3 \times 10^{-6} ; \\
& z_{\mathrm{INSTR}}=0.002231 ; u_{\mathrm{INSTR}}=0.00886 ; p_{\mathrm{INSTR}}=q_{\mathrm{INSTR}}=s_{\mathrm{INSTR}}=1 ; \\
& v_{\mathrm{INSTR}}=1.1 ; w_{\mathrm{INSTR}}=0.1 ;
\end{aligned}
$$

The value of $e_{0}$ and $e_{1}$ for each device is considered as $0.1 x$.

As we see in the above equations, the CAPEX of each IPTV component is a decreasing function of the number of subscribers $n$ and an increasing function of time $t$ because it is assumed that the CAPEX can be reduced as 
the request $(n)$ increases and can be increased for the sake of inflation as time $(t)$ evolves.

The OPEX of each IPTV component is assumed initially to be null and can increase as time and number of subscribers increase.

From research adopted in [6], the OPEX associated with Infrastructure part of the network is assumed to be a decreasing function of $m$ and an increasing function of both $n$ and $t$.

Finally, TCO for an IPTV service is:

$$
\begin{aligned}
& \mathrm{TCO}(n, m, t)=n\left(\mathrm{C}_{\mathrm{STB}}+\mathrm{O}_{\mathrm{STB}}\right)+n\left(\mathrm{C}_{\mathrm{MD} \mathrm{M}}+\mathrm{O}_{\mathrm{MDM}}\right)+\left\lceil\frac{n}{\theta}\right\rceil . \\
& \left(\mathrm{C}_{\mathrm{DSLM}}+\mathrm{O}_{\mathrm{DSLM}}+\mathrm{C}_{\mathrm{BRAS}}+\mathrm{O}_{\mathrm{BRAS}}\right)+\mathrm{C}_{\mathrm{CNT}}+2 \\
& \left(\mathrm{C}_{\mathrm{MSRVR}}+\mathrm{O}_{\mathrm{MSRVR}}\right)+m\left(\mathrm{C}_{\mathrm{ESRVR}}+\mathrm{O}_{\mathrm{ESRVR}}\right)+\mathrm{O}_{\mathrm{INSTR}}+\mathrm{O}_{\mathrm{INSTR}}
\end{aligned}
$$

Where, $\lceil x\rceil$ is the smallest integer number which is greater than or equal to $x$ and $\theta$ is the number of DSLAM ports. In this paper we have assumed that $\theta=128$.

In the current work, we have tried to track the changes which are imposed on the optimal TCO as time evolves by regulating the number of edge servers $m$ as a function of time. By work presented in [6] it is resulted that the number of edge servers can be regarded as a major parameter affecting the TCO of the IPTV solution.

Thus, the objective is to choose the optimal value of $m$ so that the TCO is minimized.

The so-called TCO minimization can be formulated as the following nonlinear programming problem [7]:

$$
m^{*}(n, t)=\underset{m \geq 0}{\arg \min } \operatorname{TCO}(n, m, t)
$$

An appropriate numerical approach is used to solve the problem for the optimal $m$ and this method leads to the time-varying minimized TCO[3]. 


\title{
4 Numerical results
}

\author{
P. Goudarzi ${ }^{*}$ M. Adeli ${ }^{+}$, M.M. Azadfar ${ }^{*}$ and F. Ayatollahi \\ - Multimedia Group, IT Faculty, Iran Telecom Research Center, Tehran-Iran \\ + Islamic Azad University of Dezful \\ Email: pgoudarzi(cuitrc.ac.ir
}

\begin{abstract}
Tin the current chapter, based on a model which is similar to that which was proposed in [6] for IPTV service deployment in Iran, a numerical analysis is performed which can find dynamically the optimal number of edge servers in terms of the number of subscribers and time. Based on the results of this numerical analysis, the service provider can optimize the TCO of its service deployment dynamically.
\end{abstract}

Keywords-TCO, CAPEX, OPEX

We have used the scenario depicted in the Fig.2 which is similar to one adopted in [6] for deployment of the IPTV service in Iran. In order to minimize the total cost (TCO) of the IPTV service, the minimization was performed with respect to $m$ (number of edge servers).

In other words, in equation 3.9 , we have to determine the optimal value of $m$ so that the total cost in this equation is minimized for a specific number of subscribers $n$ and time $t$. Time means the number of years which have elapsed since IPTV service was first deployed $(t=0)$. 
In the scenario, it is assumed that the end-users are distributed uniformly in a circular geographical region and for each ${ }_{[n / \theta]}$ user there exist a BRAS and a DSLAM. Each edge server can be connected to at least one DSLAM for streaming more demanding contents.

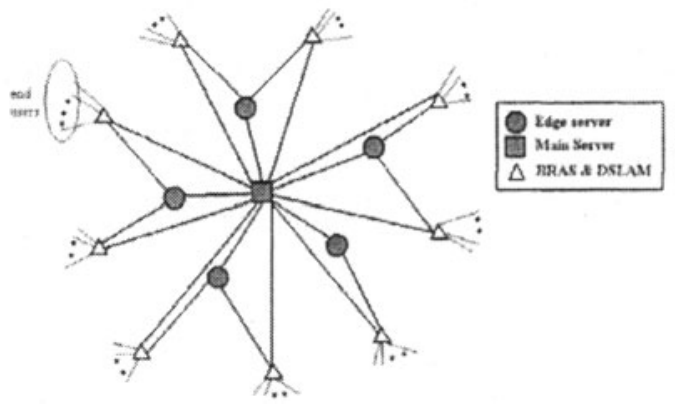

Figure 2. Sample IPTV service scenario

First, we supposed that the number of subscribers is constant and is equal to $5000(n=5000)$. Then we calculated the $m$ that minimizes the total cost at the first year of service deployment or $t=0$. The diagram of the total cost versus $m$ for $n=5000$ and $t=0$ is shown in Fig.3.

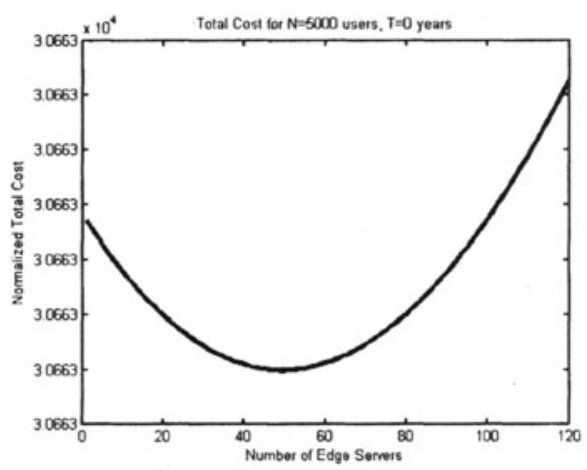

Figure 3. TCO for $n=5,000$ and $t=0$

As it is clear from Fig.3, total cost is minimum at $m=49$. To see the effect of time on the optimal $m$ (the $m$ which makes total cost minimum), we again calculated the optimal $m$ for $n=5000$ subscribers and $t=10$ years. As the Fig. 4 shows the optimal $m$ is 53 . 
The Figs.5 and 6 show the diagrams of total cost for the cases $(n=500000, t=0)$ and $(n=500000, t=10)$. Optimal $m$ in these cases is 1159 and 1163 respectively.

Figs.3-6 show that the value of $m$ which minimizes the total cost of IPTV changes as either the number of subscribers or time changes.

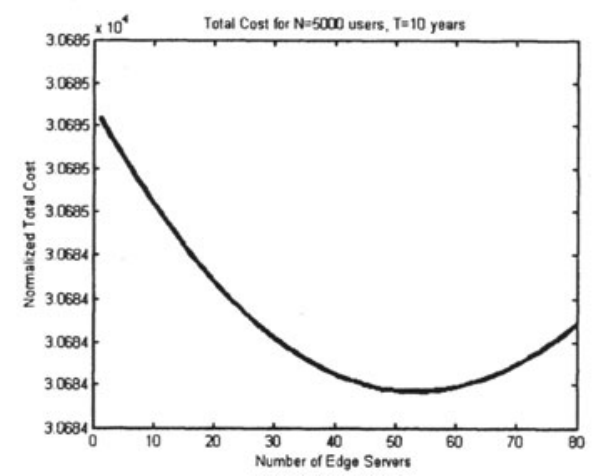

Figure 4. TCO for $n=5,000$ and $t=10$

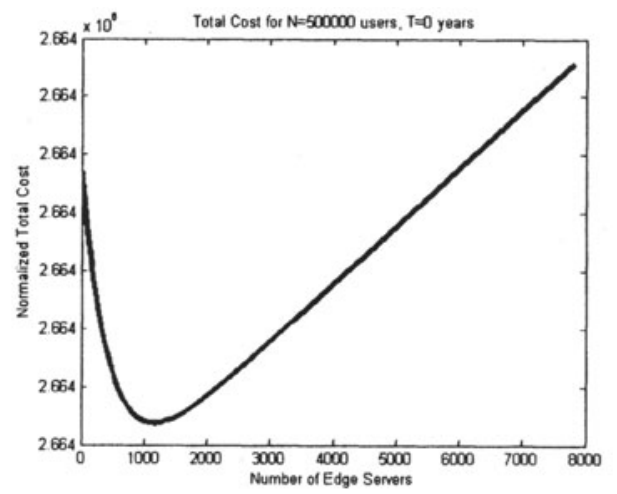

Figure 5. TCO for $n=500,000$ and $t=0$ 


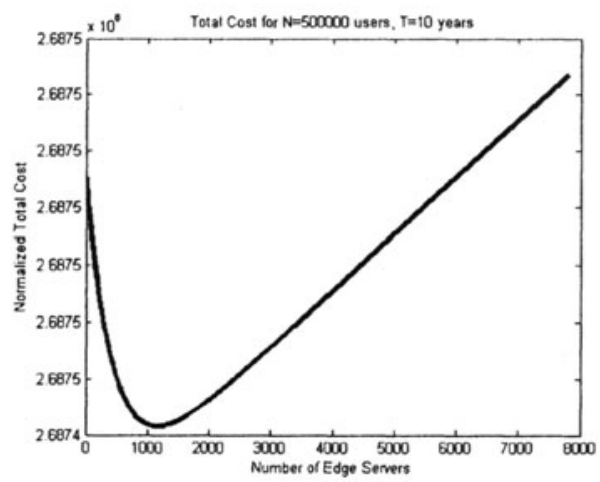

Figure 6. TCO for $n=500,000$ and $t=10$

To see the effect of time more clearly, we sketched the diagram of the total cost for $n=500000$ during the temporal period of $t=\left[\begin{array}{lllll}0 & 1 & 2 & \ldots & 20\end{array}\right]$. Fig.7 shows the result.

At last to see the changes of optimal $m$ versus time and number of subscribers, we have calculated the optimal $m$ for $n=\left[\begin{array}{lll}5000 & 1000015000 \ldots\end{array}\right.$ $1000000]$ subscribers and $t=\left[\begin{array}{lllll}0 & 1 & 2 & \ldots & 20\end{array}\right]$ years. The result is shown in Fig.8.

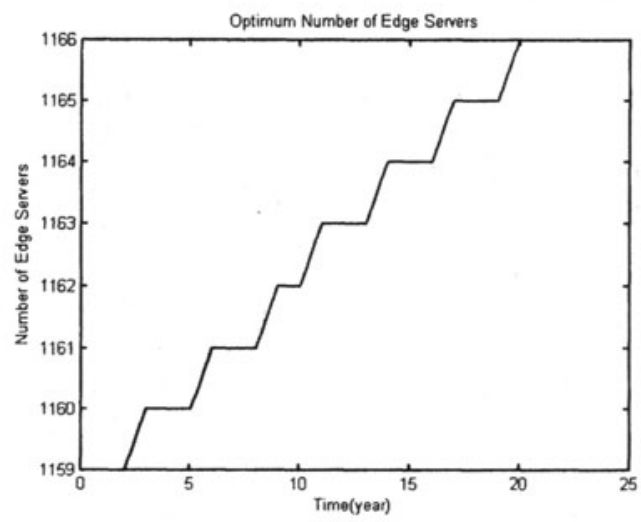

Figure 7. Optimal number of edge servers vs. time 


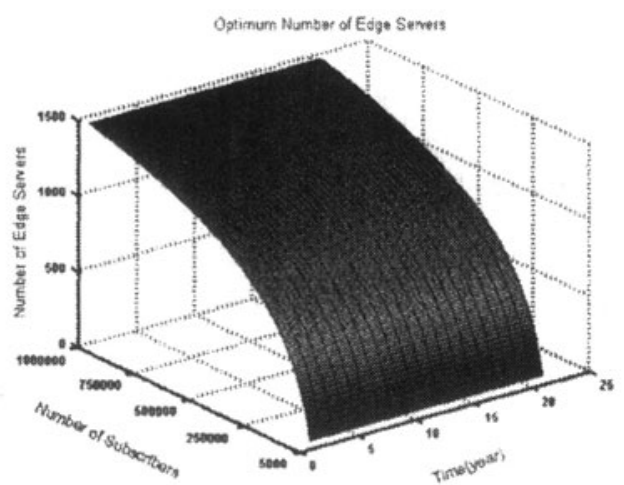

Figure 8. Optimal number of edge servers vs. time and number of subscribers

Fig.9 shows the variations occurring in the TCO of IPTV deployment with increasing time and number of subscribers.

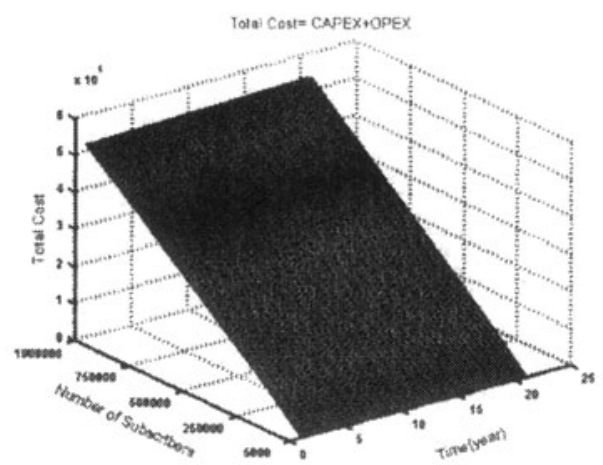

Figure 9. TCO vs. time and number of subscribers 


\section{Conclusion}

Though IPTV and VOD services are very attractive to the end users, they are highly expensive and this has caused them to be developed with a very slow pace. Minimizing the TCO (CAPEX+ OPEX) of these services is a challenge to all IPTV and VOD service providers. In an IPTV scenario one of the major factors that determine the cost of the service is the number of edge servers. We used an algorithm to minimize the TCO using selection of an optimal number of edge servers in a typical IPTV scenario. This leads to the lowest cost of service deployment. The proposed algorithm has proved to be quite efficient and dynamic in minimizing the TCO of IPTV as the number of subscribers increases and time elapses. 


\section{References}

1. Zhone technologies, In-home triple play delivery, white paper, USA, (2004).

2. Iran Telecom Research Center (ITRC), Investigating the technical, regulatory and business requirements of ACS (Advanced Communication Services) deployment in Iran, technical report, (2006).

3. D.G. Luenberger, Linear and Nonlinear Programming, 2nd Ed. Addison-Wesley Publishing Company, (1984).

4. ATIS IIF's IPTV Architecture Requirements, ATIS 0800002.

5. Internet Streaming Media Alliance (ISMA) Implementation Specification V.2 (2005).

6. Iran Telecom Research Center (ITRC), Investigating the technical aspects of developing IPTV service in IRAN, technical report, (2006).

7. D. P. Bertsekas, Nonlinear Programming: 2nd Edition. Athena Scientific, (1999). 